\title{
Threshold Upsilon-meson photoproduction at the EIC and EicC
}

\author{
Igor I. Strakovsky $\odot,{ }^{1, *}$ William J. Briscoe $\odot,{ }^{1}$ Lubomir Pentchev $\odot,{ }^{2}$ and Axel Schmidt ${ }^{1}$ \\ ${ }^{1}$ Institute for Nuclear Studies, Department of Physics, The George Washington University, \\ Washington, D. C. 20052, USA \\ ${ }^{2}$ Thomas Jefferson National Accelerator Facility, Newport News, Virginia 23606, USA
}

(Received 12 August 2021; accepted 6 October 2021; published 26 October 2021)

\begin{abstract}
High-accuracy $\Upsilon$-meson photoproduction data from EIC and EicC experiments will allow the measurement of the near-threshold total cross section of the reaction $\gamma p \rightarrow \Upsilon_{p}$, from which the absolute value of the $\Upsilon p$-scattering length, $\left|\alpha_{\Upsilon p}\right|$, can be extracted using a vector-meson dominance model. For this evaluation, we used $\Upsilon$-meson photoproduction quasidata from the QCD approach (the production amplitude can be factorized in terms of gluonic generalized parton distributions and the quarkonium distribution amplitude). A comparative analysis of $\left|\alpha_{\Upsilon p}\right|$ with the recently determined scattering lengths for $\omega p, \phi p$, and $J / \psi p$ using the A2, CLAS, and GlueX experimental data are performed. The role of the "young" vectormeson effect is evaluated.
\end{abstract}

DOI: $10.1103 /$ PhysRevD.104.074028

\section{INTRODUCTION}

In 1977, the observation of an enhancement at $9.5 \mathrm{GeV}$ in the dimuon mass spectrum produced in $400 \mathrm{GeV}$ proton-nucleus collisions at the Fermi Lab resulted in a new vector-meson $(V)$ [1], then called $\Upsilon(1 S)(9460)$.

Compared to other mesons, vector-mesons can be measured to very high precision. This stems from the fact that vector-mesons have the same quantum numbers as the photon $I^{G}\left(J^{P C}\right)=0^{-}\left(1^{--}\right)$.

The photoproduction of $J / \psi(1 S)$ and $\Upsilon(1 S)$ are among the key reactions that will be measured at the electron-ion colliders (EIC) hosted by the Brookhaven National Laboratory [2] and EicC at the High Intensity heavy-ion Accelerator Facility in China [3]. Charmonium and bottomonium are important not only for understanding the interaction mechanisms of the photoproduction of the heavy vector-mesons, but also for probing the gluonic properties of the nucleon. The large statistics of the exclusive $J / \psi(1 S)$ and $\Upsilon(1 S)$ production data at the hard scale are very helpful in probing the generalized parton distribution (GPD) of the gluon $[4,5]$. These measurements will advance our understanding of QCD which governs the properties of hadrons and the interactions involving hadrons.

\footnotetext{
*Corresponding author. igor@gwu.edu

Published by the American Physical Society under the terms of the Creative Commons Attribution 4.0 International license. Further distribution of this work must maintain attribution to the author(s) and the published article's title, journal citation, and DOI. Funded by SCOAP.
}

Exclusive vector meson photoproduction can also shed light on the bound meson-nucleon system, since the generated charmonium and bottomonium interact with an intact nucleon. We point out that the bottomonium, $\Upsilon(1 S)$, can be measured using real photons at EIC and EicC, where the quality of the expected data nearthreshold will give access to a variety of interesting physics aspects, e.g., trace anomaly, pentaquarks, cusp effects, vector-meson-nucleon scattering length, and so on. The main object of this paper is to estimate the magnitude of the absolute value of the $\Upsilon p$ scattering length, $\left|\alpha_{\Upsilon p}\right|$, using quasidata generated from the QCD model of Ref. [4], and compare it with the results for the other vector mesons. Our analysis is based also on the vector-meson dominance (VMD) model [6,7] relying on the transparent current-field identities of Kroll, Lee, and Zumino [8]. The VMD model can be used for a variety of qualitative estimates of observables in vector-meson photoproduction $[9,10]$ —at least as the first step towards their more extended theoretical studies. The use of the VMD model in case of the $J / \psi$ and $\Upsilon$ requires special attention due to the heavy mass of these vector-mesons. For the critical review of the VMD model, we refer to papers of Boreskov and Ioffe [11] and Kopeliovich et al. [12] and references therein. Recently, in Ref. [13], the VMD approach has been examined based on DysonSchwinger equations.

On the basis of recent threshold measurements of the photoproduction of three vector mesons off the proton by the A2 (MAMI), CLAS (JLab), and GlueX (JLab) Collabo rations, one can determine vector-meson-proton scattering lengths (SLs) using the VMD model [14-16]. This results in 


$$
\left|\alpha_{J / \psi p}\right| \ll\left|\alpha_{\phi p}\right| \ll\left|\alpha_{\omega p}\right|
$$

which indicates that the proton is more transparent for the $\phi$-meson compared to the $\omega$-meson, and at the same time it is much less transparent for the $\phi$-meson than for the $J / \psi$-meson. Due to the small size of "young" vector mesons relative to those that have had time to fully form, scattering lengths determined phenomenologically in the near threshold photoproduction are smaller. Recall that when the photon produces a vector meson, $V$, it first creates a $q \bar{q}$ pair in pointlike configuration. Near the threshold, this pair lacks sufficient time to form the complete wave function of the vector meson; that is, the proton interacts with the "young" (undressed) vector meson whose size is smaller than that of the "old" one participating in the elastic $V p \rightarrow V p$ scattering. Therefore, one observes stronger suppression for the vector-meson-proton interaction [17].

\section{EXCLUSIVE VECTOR-MESON PHOTOPRODUCTION AT THE EIC}

Exclusive vector-meson photoproduction is one of the key physics measurements for the EIC as discussed in the Yellow Report [2]. The proposed design for EIC is to collide electrons with energy of $E_{e}=5-18 \mathrm{GeV}$ and protons with energy of $T_{p}=41-275 \mathrm{GeV}$. The proposed EIC detector has a number of specific features that will enable photoproduction measurements, and to ensure exclusivity. First, the energy of quasireal photons will be determined by tagger detectors in the so-called "far-backward" region, i.e., in the direction of the electron beam. Tagger detectors placed approximately $24 \mathrm{~m}$ and $37 \mathrm{~m}$ from the interaction point can cover a low- $Q^{2}$ acceptance better than $10^{-7} \mathrm{GeV}^{2} / c^{2}$. Produced $\Upsilon(1 S)$ mesons will be reconstructed from their leptonic decays, e.g., to an $e^{+} e^{-}$pair. The proposed EIC detector aims for momentum resolution sufficient to cleanly separate the $\Upsilon$-states, a $\Delta p / p$ of better than $1 \%$ in the $4 \mathrm{GeV} / c-10 \mathrm{GeV} / c$ momentum range. Finally, exclusivity can be ensured by the detection of the final state proton, which, at threshold, will largely travel to the so-called "farforward" region of the detector. The far-forward region, covering angles within approximately $13 \mathrm{mrad}$ from the proton beam line, will be instrumented with a series of tracking detectors including Roman Pots, to track charged particles with slightly different magnetic rigidities than beam protons. Thus threshold $\Upsilon$ production will require the combination of the far-backward, far-forward and main EIC detectors in order to reconstruct the full event and ensure exclusivity.

\section{SCATTERING LENGTH FOR UPSILON- PROTON}

The total cross section of a binary reaction $a b \rightarrow c d$ with particle masses $m_{a}+M_{b}<m_{c}+M_{d}$ can be written as
TABLE I. Kinematical parameters for the vector-meson photoproduction off the proton at thresholds [18].

\begin{tabular}{lcrrr}
\hline \hline $\begin{array}{l}\text { Vector- } \\
\text { Meson }\end{array}$ & $\begin{array}{c}m_{V} \\
(\mathrm{MeV})\end{array}$ & \multicolumn{1}{c}{$\begin{array}{c}\sqrt{s_{\text {thr }}} \\
(\mathrm{MeV})\end{array}$} & $\begin{array}{c}E_{\text {thr }} \\
(\mathrm{MeV})\end{array}$ & \multicolumn{1}{c}{$\begin{array}{c}k_{\text {thr }} \\
(\mathrm{MeV} / c)\end{array}$} \\
\hline$\omega(782)$ & 782.65 & 1720.9 & 1109.1 & 604.7 \\
$\phi(1020)$ & 1019.461 & 1957.7 & 1573.3 & 754.0 \\
$J / \psi(1 S)$ & 3096.900 & 4035.2 & 8207.8 & 1908.5 \\
$\Upsilon(1 S)$ & 9460.30 & 10398.6 & 57152.9 & 5156.9 \\
\hline \hline
\end{tabular}

$$
\sigma_{t}=\frac{q}{k} \cdot F(q, s)
$$

where $s$ is the square of the total center of mass energy, $k$ is center of mass momentum of $a$ (and $b$ ), and $q$ is the center of mass momentum of $c$ (and $d$ ). In photoproduction, i.e., when $m_{a}=0, k=\left(s-M_{b}^{2}\right) /(2 \cdot \sqrt{s})$. Vector-meson kinematical parameters for the vector-meson photoproduction off the proton, i.e., $\gamma p \rightarrow V p$, are given in Table I and Fig. 1, where $m_{V}$ is the vector-meson mass, $s_{\text {thr }}$ is the value of $s$ at threshold, $k_{\text {thr }}$ is the value of $k$ at threshold, and $E_{\text {thr }}$ is the photon energy at threshold in the frame where the proton is initially at rest.

The factor $F(q, s)$ in Eq. (2) is proportional to the square of the invariant amplitude and does not vanish at threshold, i.e., when $q \rightarrow 0$ and $k \rightarrow k_{\text {thr }}$, but instead approaches a constant value. Thus, near threshold, $\sigma_{t} \rightarrow 0$ and is at least proportional to $q$.

Traditionally, the $\sigma_{t}$ behavior of a near-threshold binary inelastic reaction is described as a series of odd powers in $q$ (see, for instance, Ref. [14]). In the energy range under study, we use

$$
\sigma_{t}(q)=b_{1} \cdot q+b_{3} \cdot q^{3}+b_{5} \cdot q^{5}
$$

which assumes contributions from only the lowest $S$-, $P$-, and $D$-waves. Very close to threshold, the higher-order terms can be neglected and the linear term is determined by the $S$-wave only with a total spin of $1 / 2$ and/or $3 / 2$.

For the evaluation of the absolute value of the vectormeson-proton SL, we apply the commonly used and effective VMD approach (Fig. 2), which links the nearthreshold cross sections of the vector-meson photoproduction, $\gamma p \rightarrow V p$, and the elastic scattering, $V p \rightarrow V p$, processes via [10]

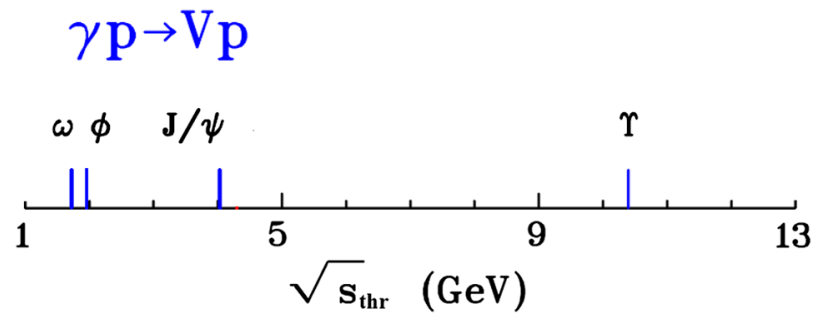

FIG. 1. Thresholds of meson photoproduction off the proton. Blue vertical lines are for vector-mesons including charmonium and $b$-quarkonium. 


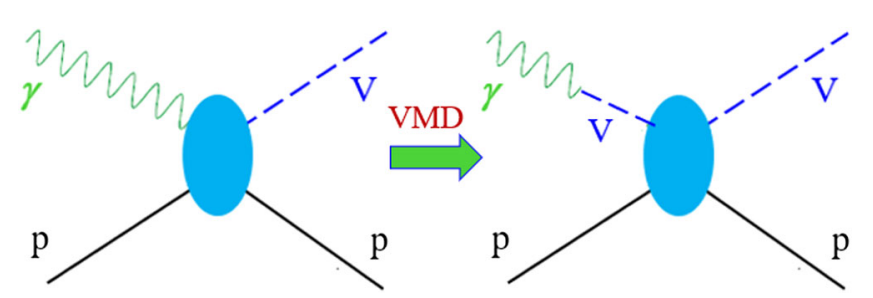

FIG. 2. Schematic diagrams of vector-meson photoproduction (left) and the VMD model (right) in the energy region at threshold experiments.

$$
\begin{aligned}
\left.\frac{d \sigma^{\gamma p \rightarrow V p}}{d \Omega}\right|_{\mathrm{thr}} & =\frac{q}{k} \cdot \frac{1}{64 \pi} \cdot\left|T^{\gamma p \rightarrow V p}\right|^{2} \\
& =\left.\frac{q}{k} \cdot \frac{\pi \alpha}{g_{V}^{2}} \cdot \frac{d \sigma^{V p \rightarrow V p}}{d \Omega}\right|_{q \rightarrow 0} \\
& =\frac{q}{k} \cdot \frac{\pi \alpha}{g_{V}^{2}} \cdot\left|\alpha_{V p}\right|^{2}
\end{aligned}
$$

where $T^{\gamma p \rightarrow V p}$ is the invariant amplitude of the vector-meson photoproduction, $\alpha$ is the fine-structure constant, and $g_{V}$ is the VMD coupling constant, related to the vector-meson electromagnetic (EM) decay width $\Gamma_{V \rightarrow e^{+} e^{-}}$

$$
g_{V}^{2}=\frac{\pi \cdot \alpha^{2} \cdot m_{V}}{3 \cdot \Gamma\left(V \rightarrow e^{+} e^{-}\right)},
$$

where $m_{V}$ is the vector-meson mass.

Combining Eq. (3) (which is also valid for $\omega$ - $\phi$-, and $J / \psi$-photoproduction [14-16]) and Eq. (4) with Eq. (5), one can express the absolute value of the SL as a product of the pure EM, VMD-motivated kinematic factor

$$
B_{V}^{2}=\frac{\alpha \cdot m_{V} \cdot k}{12 \pi \cdot \Gamma\left(V \rightarrow e^{+} e^{-}\right)},
$$

and the factor

$$
h_{V p}=\sqrt{b_{1}}
$$

that is determined by an interplay of strong (hadronic) and EM dynamics as

$$
\left|\alpha_{V p}\right|=B_{V} \cdot h_{V p} .
$$

Numerical values of both $g_{V}$ and $B_{V}$ are given in Table II. Let us note that these EM quantities $B_{V}$ for each vectormeson are close to each other except for $\Upsilon$.

Figure 3 shows the fit of the QCD model of Ref. [4] to the GlueX $J / \psi$ photoproduction data [19] and the prediction of this model for the $\Upsilon$ photoproduction total cross section. Our phenomenological fit of the GlueX data using Eq. (3) is shown, as well. This is a rather general model assuming factorization in terms of gluonic generalized parton distributions and quarkonium wave function on
TABLE II. Vector-meson EM properties. The decay $\Gamma(V \rightarrow$ $e^{+} e^{-}$) from PDG2020 [18] (second column), $g_{V}$ was calculated using Eq. (5) (third column) and $B_{V}$ using Eq. (6) (fourth column).

\begin{tabular}{lccc}
\hline \hline $\begin{array}{l}\text { Vector- } \\
\text { Meson }\end{array}$ & $\begin{array}{c}\Gamma\left(V \rightarrow e^{+} e^{-}\right) \\
(\mathrm{keV})\end{array}$ & $g_{V}$ & $\begin{array}{c}B_{V} \\
\left(\mathrm{MeV}^{1 / 2}\right)\end{array}$ \\
\hline$\omega(782)$ & $0.60 \pm 0.02$ & $8.53 \pm 0.14$ & $390.49 \pm 6.35$ \\
$\phi(1020)$ & $1.27 \pm 0.04$ & $6.69 \pm 0.10$ & $342.50 \pm 5.27$ \\
$J / \psi(1 S)$ & $5.53 \pm 0.10$ & $5.59 \pm 0.05$ & $454.92 \pm 4.06$ \\
$\Upsilon(1 S)$ & $1.340 \pm 0.018$ & $19.85 \pm 1.21$ & $2654.96 \pm 162.15$ \\
\hline \hline
\end{tabular}

one side and hard quark-gluon interaction on the other side. This work extends the validity of the factorization, as studied previously in Ref. [5] for high energies, down to the threshold region in leading order and in the case of a heavy quark mass.

We have generated quasidata (Fig. 4) for the $\Upsilon$ cross sections from this model [4] and fit it with Eq. (3) in order to extrapolate the data to the threshold. The exact $q_{\text {min }}$ attainable in an ep collider experiment will depend heavily on the exact placement of the low- $Q^{2}$ tagging detectors. For the purposes of this paper, we assume that the detector will have comparable coverage to the EIC Yellow Report detector [2], where the backward calorimeter coverage down to a pseudorapidity of -4 would allow $q_{\text {min }}$ for $\Upsilon$ photoproduction to be as low as $\approx 500 \mathrm{MeV} / \mathrm{c}$. Further optimization of the low- $Q^{2}$ taggers may allow an even smaller $q_{\text {min }}$ to be achieved. Gryniuk et al. assumed a total integrated luminosity of $100 \mathrm{fb}^{-1}$ for the $\Upsilon$ photoproduction at EIC, which corresponds to

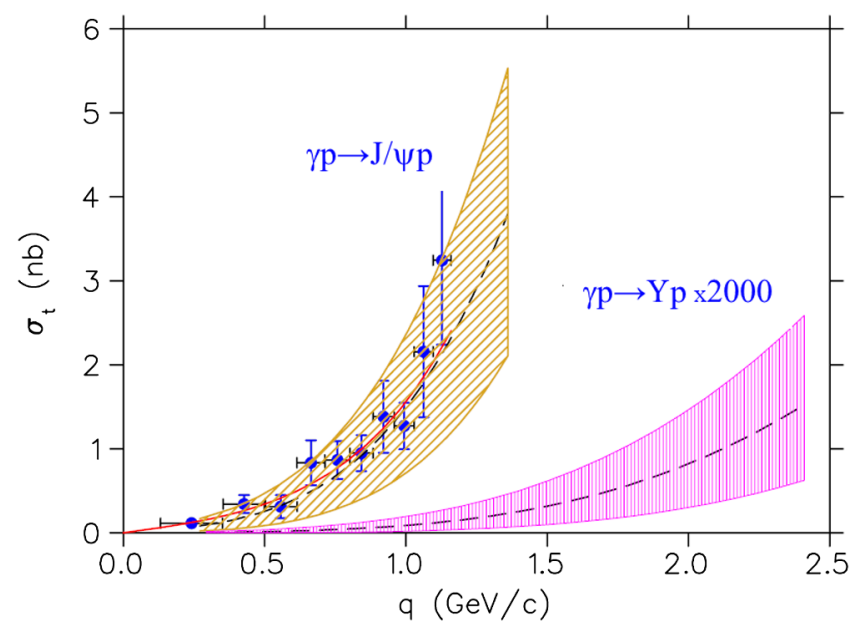

FIG. 3. The total $\gamma p \rightarrow J / \psi p$ and $\gamma p \rightarrow \Upsilon p$ cross sections $\sigma_{t}$. GlueX threshold $J / \psi$ photoproduction data [19] shown by blue filled circles and the best-fit results are from Ref. [16] and shown by red solid curve. Theoretical fit of the GlueX data from Ref. [4] is shown by yellow shaded band at 95\% C.L. Theoretical predictions for $\Upsilon$ photoproduction [4] are shown by magenta shaded band. 


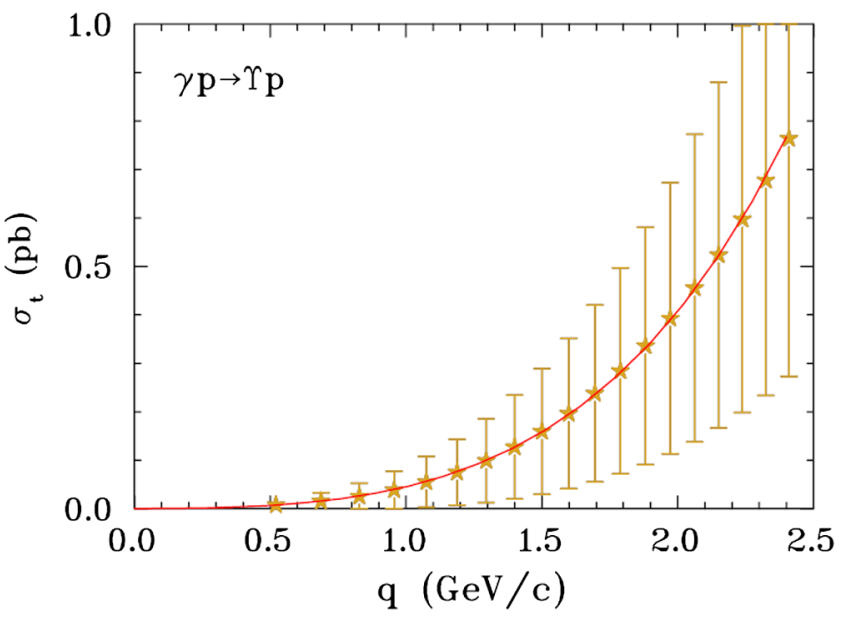

FIG. 4. The total $\gamma p \rightarrow J / \psi p$ cross sections $\sigma_{t}$. Quasidata from Ref. [4] $\left(q_{\min }=521 \mathrm{MeV} / c\right)$ shown by yellow filled stars The phenomenological best fit for a quasi cross section [4] is shown by red solid curve. The uncertainties for quasidata were taken from Ref. [4].

116 days of the beam with the $10^{34} \mathrm{~cm}^{-2} \mathrm{~s}^{-1}$, for Monte Carlo calculations [20]. As Guo et al. [4] pointed out, the large mass of the $\Upsilon$-meson implies that the calculations in the heavy meson limit works better for the $\Upsilon$ photoproduction, as there are fewer theoretical uncertainties from higher-order corrections. We account here for the theoretical accuracy and do not include the statistical/experimental errors. The expected luminosity of $10^{34} \mathrm{~cm}^{-2} \mathrm{~s}^{-1}$ will be able to provide smaller statistical uncertainty than the theoretical one. However, as the parameters of the EIC detector have not been defined yet, this estimate will depend on the actual detector acceptance and run time. Figure 5 illustrates the dramatic differences in the hadronic factors $h_{V p}=\sqrt{b_{1}}$ (see Table III), as the slopes $\left[b_{1}\right.$ from Eq. (3)] of the total cross sections at threshold as a function of $q$ vary significantly from $\omega$ to $J / \psi$ and now to $\Upsilon$.

Therefore, such a big difference in SLs of the vectormeson-proton systems is determined mainly by the hadronic factor $h_{\underline{V} p}$, and reflects a strong weakening of the interaction in the $\bar{b} b-p$ and $\bar{c} c-p$ systems compared to that of the light $\bar{q} q-p(q=u, d)$ configurations. The interaction in the $\bar{s} s-p$ has an intermediate strength that is manifested in an intermediate value of the $\phi p$ SL.

The corresponding results for the scattering lengths are shown in Fig. 6 as a function of the inverse vector-meson mass. Starting from the $\phi$ meson and for higher masses, the scattering lengths are significantly smaller than the typical hadron size of $1 \mathrm{fm}$, indicating increasing transparency of the proton for these mesons. Moreover, our analysis shows almost linear (in exponential scale) increase $\left|\alpha_{V p}\right| \propto \exp \left(1 / m_{V}\right)$ with increasing $1 / m_{V}$. Actually, $p \rightarrow V$ coupling is proportional to the strong coupling $\alpha_{s}$ and the separation of the corresponding

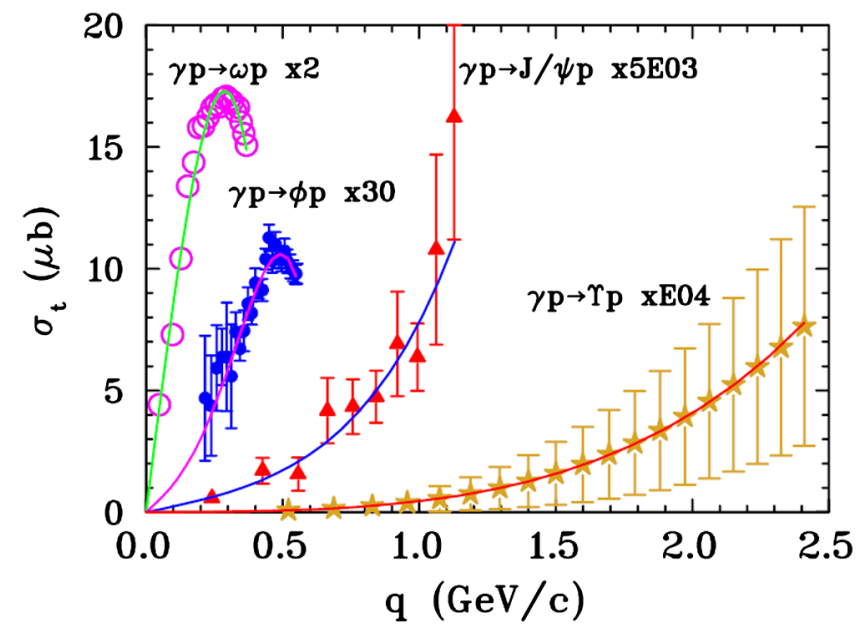

FIG. 5. The total $\gamma p \rightarrow V p$ cross section $\sigma_{t}$ derived from the A2 (magenta open circles) [14], CLAS (blue filled circles) [15], and GlueX (red filled triangles) [16], data, and EIC/EicC (yellow filled stars) quasidata is shown as a function of the center of mass momentum $q$ of the final-state particles. The vertical (horizontal) error bars represent the total uncertainties of the data summing statistical and systematic uncertainties in quadrature (energy binning). Solid curves are the fit of the data with Eq. (3).

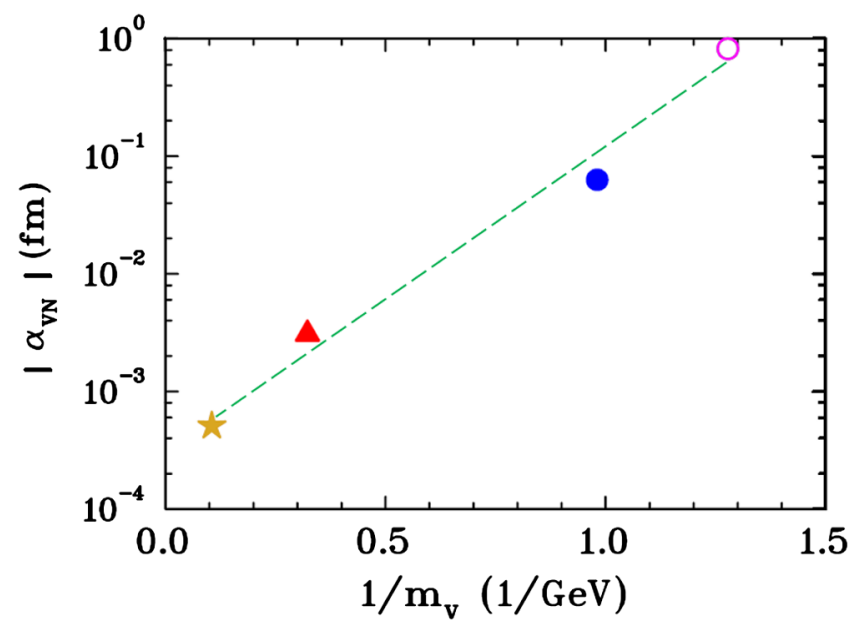

FIG. 6. Comparison of the $\left|\alpha_{V p}\right|$ SLs estimated from vectormeson photoproduction at threshold vs the inverse mass of the vector mesons. The magenta open circle shows the analysis of the A2 $\omega$-meson data [14], the blue-filled circle shows the analysis of the CLAS $\phi$-meson data [15], the red-filled triangle shows the analysis of the GlueX $J / \psi$-meson data [16], and the yellow-filled star shows the analysis of the EIC and EicC $\Upsilon$-meson quasidata [4]. The green dashed line is hypothetical.

quarks. This separation (in zero approximation) is proportional to $1 / m_{V}$, which may explain the above relation.

\section{DISCUSSIONS AND OUTLOOK}

In summary, one can extend the relationship (1) including the result for $\Upsilon$ (Table III) 
TABLE III. The second column showed the minimal momentum $q_{\text {min }}$ for vector-mesons in photoproduction experiments and the source of data. The linear term (third column) of the best fit of the total cross section data using Eq. (3). The errors represent the total uncertainties (summing statistical and systematic uncertainties in quadrature. The fourth column showed vector-mesonproton SLs.

\begin{tabular}{lccc}
\hline \hline $\begin{array}{l}\text { Vector- } \\
\text { Meson }\end{array}$ & $\begin{array}{c}q_{\min } \\
(\mathrm{MeV} / c)\end{array}$ & $\begin{array}{c}b_{1} \\
(\mu b / \mathrm{MeV} / c)\end{array}$ & $\begin{array}{c}\left|\alpha_{V p}\right| \\
(\mathrm{fm})\end{array}$ \\
\hline$\omega(782)$ & $49[14]$ & $(0.44 \pm 0.01) \times 10^{-1}[14]$ & $0.82 \pm 0.03[14]$ \\
$\phi(1020)$ & $216[21]$ & $(0.34 \pm 0.12) \times 10^{-3}[15]$ & $0.063 \pm 0.010[15]$ \\
$J / \psi(1 S)$ & $230[19]$ & $(0.46 \pm 0.16) \times 10^{-6}[16]$ & $(3.08 \pm 0.55) \times 10^{-3}[16]$ \\
$\Upsilon(1 S)$ & $521[4]$ & $(0.37 \pm 0.04) \times 10^{-9}$ & $(0.51 \pm 0.03) \times 10^{-3}$ \\
\hline \hline
\end{tabular}

$$
\left|\alpha_{\Upsilon p}\right| \ll\left|\alpha_{J / \psi p}\right| \ll\left|\alpha_{\phi p}\right| \ll\left|\alpha_{\omega p}\right| .
$$

The values of $\left|\alpha_{V p}\right|$ for the heavy vector-mesons, as determined in the recent papers [14-16] using the VMD model, are smaller than most of the theoretical predictions; see references in Refs. [14-16]. As for the $\Upsilon$ case, we are not aware of any theoretical predictions for the $\Upsilon_{p}$ scattering length except, related to that, a nonrelativistic potential result for the radius of the bottominium which is $r_{\Upsilon}=(0.14 \pm 0.0014) \mathrm{fm}[22]$. The same approach gives a radius of the charmonium of $r_{J / \psi}=(0.25 \pm 0.0025) \mathrm{fm}$. Gryniuk et al. reported recently $\left|\alpha_{\Upsilon p}\right|=(0.066 \pm 0.001)$ fm and $\left|\alpha_{\Upsilon p}\right|=(0.016 \pm 0.001)$ fm [20] for two different subtraction constants. The latter results are based on the dispersive relations, taking some assumptions from their $J / \psi$ photoproduction model [23], and extrapolating the high-energy measurements at $W \approx 100 \mathrm{GeV}$ down to the threshold.

The smallness of the scattering lengths, as extracted with the help of the VMD model, can be related to the "young age" of the vector mesons participating in the interaction with the proton as introduced by Feinberg [17]. For more quantitative estimate of the theoretical uncertainty related to the VMD model, we refer to the paper by Boreskov and Ioffe [11]. They evaluated the cross section of $J / \psi$ photoproduction in a peripheral model (a mechanism for the photoproduction of $J / \psi$ off nucleon with an imaginary amplitude that arises as the shadow of the amplitudes for the photoproduction of charmed particles) by Ref. [11] and found a strong energy dependence because the nondiagonal process $\gamma p \rightarrow J / \psi p$ must have larger transfer momenta versus the elastic scattering $J / \psi p \rightarrow J / \psi p$. This result is in a violation of VMD by a factor of five or so. In the case of the $\Upsilon$ meson, we expect the disagreement to be even worse. Boreskov et al. showed that a fluctuation of a photon into open charm particles is preferable than into charmonium [24]. Later on, it was shown by Kopeliovich et al. that the open-charm cross section is larger than the charmonium one by a factor of ten or more $[25,26]$. In addition, in Eq. (4), we did not include a factor introduced in the VMD model in Ref. [27], which takes into account the difference between polarization degrees of freedom in the $\gamma p \rightarrow J / \psi p$ and $p J / \psi \rightarrow p J / \psi$ reactions. Such a factor that equals $2 / 3$ at threshold for the $S$-wave has not been used in the previous analysis of the scattering lengths; we consider it as a systematic uncertainty related to the VMD model [16].

In the recent work of Ref. [13] the effect of the VMD assumption was studied in the formalism of the DysonSchwinger equations which one can consider as an alternative interpretation of the "young age" effect in another (more formal) language. Their result shows much dramatic effect, up to a factor of 50 overestimation of the cross sections when using VMD for the two heaviest vector mesons. Nevertheless, this translates into a factor of seven for the scattering lengths and the overall dependence in Fig. 6 remains similar.

Present and future experiments JLab, EIC, and EicC that are aimed to measure charmonium and bottomonium production on the proton and nuclei will allow further studies of $J / \psi N$ and $\Upsilon N$ interactions and will allow access to a variety of other interesting physics aspects that are present in the near-threshold region. Further studies on both nucleons and nuclei in heavy vector-meson photo and electroproduction will significantly extend our knowledge of the gluonic structure of the nuclear matter.

\section{ACKNOWLEDGMENTS}

We thank Misha Ryskin for useful remarks and continuous interest in the paper, Yulia Furletova for the detector design for the EIC, and Yuxun Guo for predictions of $\Upsilon$-photoproduction cross sections. This work was supported in part by the U.S. Department of Energy, Office of Science, Office of Nuclear Physics under Award No. DE-SC0016583 and Contract No. DE-AC05-06OR23177. 
[1] S. W. Herb et al., Observation of a Dimuon Resonance at 9.5-GeV in 400-GeV Proton-Nucleus Collisions, Phys. Rev. Lett. 39, 252 (1977).

[2] R. A. Khalek et al. Science requirements and detector concepts for the electron-ion collider: EIC Yellow Report, arXiv:2103.05419.

[3] D. P. Anderle et al. Electron-ion collider in China, Front. Phys. (Beijing) 16, 64701 (2021).

[4] Y. Guo, X. Ji, and Y. Liu, QCD analysis of near-threshold photon-proton production of heavy quarkonium, Phys. Rev. D 103, 096010 (2021).

[5] D. Y. Ivanov, A. Schafer, L. Szymanowski, and G. Krasnikov, Exclusive photoproduction of a heavy vector meson in QCD, Eur. Phys. J. C 34, 297 (2004); Erratum, Eur. Phys. J. C 75, 75 (2015).

[6] M. Gell-Mann and F. Zachariasen, Form-factors and vector mesons, Phys. Rev. 124, 953 (1961).

[7] J. J. Sakurai, Currents and Mesons (The University of Chicago Press, Chicago, 1969).

[8] N. M. Kroll, T. D. Lee, and B. Zumino, Neutral vector mesons and the hadronic electromagnetic current, Phys. Rev. 157, 1376 (1967).

[9] M. F. M. Lutz, G. Wolf, and B. Friman, Scattering of vector mesons off nucleons, Nucl. Phys. A706, 431 (2002); Erratum, Nucl. Phys. A765, 431 (2006); Erratum, Nucl. Phys. A765, 495 (2006).

[10] A. I. Titov, T. Nakano, S. Date, and Y. Ohashi, Comments on differential cross-section of phi-meson photoproduction at threshold, Phys. Rev. C 76, 048202 (2007).

[11] K. G. Boreskov and B. L. Ioffe, $J / \psi$ meson photoproduction in the peripheral model, Yad. Fiz. 25, 806 (1977) [Sov. J. Nucl. Phys. 25, 331 (1977)].

[12] B. Z. Kopeliovich, I. Schmidt, and M. Siddikov, Suppression versus enhancement of heavy quarkonia in pA collisions, Phys. Rev. C 95, 065203 (2017).

[13] Y.Z. Xu, S. Chen, Z. Q. Yao, D. Binosi, Z. F. Cui, and C. D. Roberts, Vector-meson production and vector meson dominance, arXiv:2107.03488.

[14] I. I. Strakovsky et al. (A2 Collaboration at MAMI), Photoproduction of the $\omega$ meson on the proton near threshold, Phys. Rev. C 91, 045207 (2015).
[15] I. Strakovsky, L. Pentchev, and A. Titov, Comparative analysis of $\omega p, \phi p$, and $J / \psi p$ scattering lengths from A2, CLAS, and GlueX threshold measurements, Phys. Rev. C 101, 045201 (2020).

[16] I. Strakovsky, D. Epifanov, and L. Pentchev, J/ $\psi$-p scattering length from GlueX threshold measurements, Phys. Rev. C 101, 042201 (2020).

[17] E. L. Feinberg, Hadron clusters and half dressed particles in quantum field theory, Usp. Fiz. Nauk 132, 225 (1980) [Sov. Phys. Usp. 23, 629 (1980)].

[18] P. A. Zyla et al. (Particle Data Group Collaboration), Review of particle physics, Prog. Theor. Exp. Phys. 2020, 083C01 (2020).

[19] A. Ali et al. (GlueX Collaboration), First Measurement of Near-Threshold $J / \psi$ Exclusive Photoproduction off the Proton, Phys. Rev. Lett. 123, 072001 (2019).

[20] O. Gryniuk, S. Joosten, Z. E. Meziani, and M. Vanderhaeghen, $\Upsilon$ photoproduction on the proton at the electron-ion collider, Phys. Rev. D 102, 014016 (2020).

[21] B. Dey et al. (CLAS Collaboration), Data analysis techniques, differential cross sections, and spin density matrix elements for the reaction $\gamma p \rightarrow \phi p$, Phys. Rev. C 89, 055208 (2014).

[22] H. Satz, Quarkonium binding and dissociation: The spectral analysis of the QGP, Nucl. Phys. A783, 249 (2007).

[23] O. Gryniuk and M. Vanderhaeghen, Accessing the real part of the forward $J / \psi-$ p scattering amplitude from $J / \psi$ photoproduction on protons around threshold, Phys. Rev. D 94, 074001 (2016).

[24] K. Boreskov, A. Capella, A. Kaidalov, and J. Tran Thanh Van, Heavy quark and lepton pair production on nuclei, Phys. Rev. D 47, 919 (1993).

[25] J. Hufner, Y. Ivanov, B. Kopeliovich, and A. Tarasov, Photoproduction of charmonia and total charmonium proton cross-sections, Phys. Rev. D 62, 094022 (2000).

[26] B. Floter, B. Kopeliovich, H. Pirner, and J. Raufeisen, Charmed meson production in deep inelastic scattering, Phys. Rev. D 76, 014009 (2007).

[27] V. Kubarovsky and M. B. Voloshin, Formation of hiddencharm pentaquarks in photon-nucleon collisions, Phys. Rev. D 92, 031502 (2015). 